Original Research Article

\title{
Antimicrobial usage and its quantification for neonatal sepsis at a tertiary care hospital neonatal intensive care unit
}

\author{
Manjari Kumari $^{1}$, Suparna Chatterjee ${ }^{2 *}$, Tapas Som $^{3}$, Pinaki Chattopadhyay ${ }^{4}$
}

\begin{abstract}
${ }^{1}$ Department of Pharmacology, Rajshree Medical Research Institute, Bareilly, Uttar Pradesh, India ${ }^{2}$ Department of Pharmacology, Institute of Postgraduate Medical Education \& Research , Kolkata, West Bengal, India ${ }^{3}$ Department of Neonatology, All India Institute of Medical Sciences Bhubaneswar, Odisha, India

${ }^{4}$ Department of Neonatal Microbiology, Institute of Postgraduate Medical Education \& Research and SSKM Hospital Kolkata, West Bengal, India
\end{abstract}

Received: 04 June 2019

Revised: 29 June 2019

Accepted: 16 July 2019

\section{*Correspondence to:}

Dr. Suparna Chatterjee, Email: drsupchat@gmail.com

Copyright: (C) the author(s), publisher and licensee Medip Academy. This is an openaccess article distributed under

\begin{abstract}
Background: Antimicrobial therapy for neonatal sepsis is challenging as its judicious use can save neonates while its inappropriate use can lead to rapid emergence of resistant strains. Quantification of consumption of antimicrobial agents (AMA) has not been undertaken in Indian neonatal intensive care units (NICU) setting. This prospective observational study evaluated the antimicrobial prescribing pattern and quantified its consumption in clinically suspected neonatal sepsis (NS) cases.

Methods: Clinically suspected NS cases admitted over study period of 18 months in a tertiary care level III NICU were enrolled. Data of antimicrobials prescribed, its consumption, culture sensitivity profile of organisms isolated were collected.Consumption was quantified by computing the days of therapy (DOT) per 1000 patient-days (PD).

Results: 150 clinically suspected NS cases were enrolled; $37.33 \%$ were culture positive. The most common AMA prescribed were netilmicin (94.67\%), piperacillin-tazobactam $(88.67 \%)$. Only $0.67 \%$ cases received reserve antimicrobials like colistin, vancomycin and linezolid. 58\% received 2 AMA, $39.33 \%$ received $\geq 3$ agents. Total antimicrobial consumption was 614.86 DOT/1000 PD and 21.68 DOT/ neonate. Statistically significant difference in total AMA consumption amongst culture positive versus negative cases ( $p$ $<0.001)$ was observed but difference was not significant in EOS versus LOS $(\mathrm{p}=0.95)$.

Conclusion: Usage of antimicrobials for neonatal sepsis was guided by sensitivity pattern of local prevalent flora and clinical response. Usage of reserve antimicrobials were restricted. However, consumption of $\mathrm{AB}$ was higher compared to developed countries and we intend to use the study outcome data for antibiotic stewardship program to reduce antibiotic consumption and modify prescribing trends at the study setting.
\end{abstract}

Key words: Antimicrobials, Antibiotic usage, Days of therapy, Neonatal sepsis, Neonatal intensive care unit

\section{INTRODUCTION}

Neonatal sepsis is a major cause of morbidity and mortality worldwide especially in developing countries. Published reports from the National Neonatal and Perinatal database of India, estimated the incidence of neonatal sepsis at $30 / 1000$ live births ${ }^{1}$ while other epidemiological studies have suggested a rate varying from 49-170 per 100 live births. ${ }^{2}$ The commonest cause of neonatal mortality is sepsis, accounting for about 30$50 \%$ of the total neonatal deaths in developing countries. ${ }^{3,4}$ Antibiotics are one of the most commonly prescribed category of medications in the NICU. ${ }^{5}$ The use of antimicrobials in neonatal sepsis is challenging as its judicious use saves life while its inappropriate and irrational use can lead to rapid emergence of infections by drug resistant strains and increased likelihood of other antimicrobial associated related adverse outcomes in neonates.

Clinicians initiate empirical antimicrobial therapy based on strong clinical suspicion but often find it difficult to 
stop it despite culture negative reports. Culture-negative sepsis accounts for about 10 to $30 \%$ of total antibiotic consumption. ${ }^{6,7}$ Appropriate selection of AMA in NS is a major challenge for clinicians since the clinical features are often subtle coupled with fear of aggravation of the suspected clinical condition thereby culminating in a natural tendency for over prescribing antibiotics which has often lead to the rapid emergence of antibiotic resistant strains. An overall rise in healthcare costs, lack of uniformity in drug prescribing and the emergence of antibiotic resistance, monitoring and control of antibiotic use are of growing concern and strict antibiotic policies are warranted. Few neonatal intensive care units (NICU) in India strictly follow specific guidelines for antibiotic usage in neonatal sepsis while majority of them practice empiric therapy. Evidence based judicious use of antibiotics is an important means to limit the emergence of antibiotic-resistant organisms.

One of the important means of advocating rational antimicrobial usage in NICU setting is by formulating and implementing an "Antibiotic Stewardship Program". An important prerequisite for developing an antibiotic stewardship program includes characterizing the antimicrobial usage pattern of the setup and its in-depth analysis, identifying patients who need antibiotic therapy, using local epidemiology to guide the selection of empirical therapy, avoiding agents with overlapping activity, adjusting antibiotics when culture results become available, monitoring for toxicity, and optimizing the dose, route, and duration of therapy.

Published data regarding antimicrobial usage and its quantification in neonatal sepsis is limited from India. Hence, the study objectives were to evaluate the antimicrobial prescribing patterns and quantification of its consumption in a level 3 NICU setting of a tertiary care teaching hospital of eastern India.

\section{METHODS}

The study was a prospective longitudinal observational study conducted at the NICU of a tertiary care hospital in eastern India for a period of 18 months (from February 2015 to August 2016). Study was initiated after getting approval from Institutional Ethics Committee. Since this was a time bound observational study, no formal sample size calculation was done. Potential study subjects were screened and babies who were admitted in NICU with clinically suspected neonatal sepsis, receiving systemic antimicrobial (antibiotic/antifungal/antiviral) agents were included. Babies receiving antimicrobial agents due to indications other than neonatal sepsis like prophylactic antibiotic use for surgery were excluded from the study.

Information pertaining to use of all antimicrobials administered was collected in a predesigned structured case record form (CRF). Detailed information about the baby included mode of delivery, place of delivery, presence or absence of any maternal risk factors for sepsis, birth weight, gestational age, type of sepsis early onset sepsis (EOS) or late onset sepsis (LOS), duration of NICU stay and treatment outcome. EOS was considered if sepsis presents within 72 hours of birth and LOS $\geq 72$ hours of age.

Data of antimicrobial usage included the prescribed dose, route of administration, frequency, reason (s) for initiation, discontinuation, and duration of use of each agent. Sepsis screen reports which included total leukocyte count, absolute neutrophil count, immature to total neutrophil ratio, micro-erythrocyte sedimentation rate and $\mathrm{C}$ reactive protein were noted.

Identification and susceptibility pattern of organisms isolated from biological samples (blood, CSF, urine, peritoneal aspirate and tracheal aspirate) were also collected. Blood and CSF culture was done in the microbiology lab by BACTEC method and sensitivity testing was done by using Disc diffusion method (Kirby Bauer method). Neonates were followed till the outcome (death, discharge, transferred out of the unit or discharged against medical advice) and the length of stay in hospital was noted.

Quantification of antimicrobial consumption was computed and expressed as days of therapy (DOT) metrics. DOT is an aggregate sum of the days of exposure for each antimicrobial agent and was determined by multiplying the number of antibiotic doses by the dosing interval, then dividing by 24 hours. $^{8}$

There is limited published data regarding usage of DOT as a measure of quantification of antimicrobial agents in NICU setting in developing countries. Most of the studies have reported the data in terms of proportions of antimicrobial agents. In adults as per the recommendation of Infectious Diseases Society of America (IDSA) the World Health Organization (WHO) "Defined daily dose" per 1000 patient days (DDD/1000 PD) and Days of Therapy per 1000 patient days (DOT/ $1000 \mathrm{PD}$ ) are to be used as the measurement unit for antimicrobials. ${ }^{9}$

However, the WHO "DDD" can be used only for adult drug usage and cannot be used for paediatric or neonatal population. Therefore, DOT is a better metric for antimicrobial agents use in paediatric patients. ${ }^{10}$

One DOT represents the administration of a single agent on a given day regardless of the number of doses administered or dosage strength; in essence, 1 DOT is the administration of at least one dose of a single agent on a given day. The length of stay for all neonates during the study period was summed to determine the total number of patient days.

DOT was adjusted for hospital census, in terms of per 1,000 patient days (PD). Expression of data in terms of per 1000 patient days normalizes antimicrobial use data 
to account for fluctuations in patient census and length of stay.

\section{Statistical analysis}

Statistical analysis was done by SPSS (Statistical Package for Social Sciences, version 16.0.1 of IBM, USA) and GraphPad Prism Version 5.0 of GraphPad Software, USA statistical soft wares. All study variables depending on the data type were summarized using appropriate measures of central tendency (mean, median) and dispersion - standard deviation (SD) or interquartile range (IQR). Categorical variables were expressed as frequencies and percentages. Inferential statistics for comparing data between various subgroups was done either by Student's unpaired t test for normally distributed numerical variables, or by Mann-Whitney U test for non-parametric variables. Fisher's exact test or Pearson's Chi-square test was done for comparison of categorical variables. Odd's Ratio was computed for determining association between variables. Value of $\mathrm{p} \leq 0.05$ were considered statistically significant along with the $95 \%$ confidence interval for the test statistic was computed.

\section{RESULTS}

During the study period, 150 clinically suspected NS cases were enrolled. The baseline characteristics of the study population are presented in Table 1 .

Table 1: Baseline characteristic profile of enrolled subjects.

\begin{tabular}{|c|c|}
\hline Characteristic (n=150) & Value \\
\hline $\begin{array}{l}\text { Gestational age in week } \\
(\text { Mean } \pm \text { SD) }\end{array}$ & $33.29 \pm 3.81$ \\
\hline Birth weight in g (Mean \pm SD) & $1614 \pm 770.89$ \\
\hline \multicolumn{2}{|l|}{ Gender N (\%) } \\
\hline Male & $90(60)$ \\
\hline Female & $60(40)$ \\
\hline \multicolumn{2}{|l|}{ Gestational category N (\%) } \\
\hline$<37$ weeks & $112(74.67)$ \\
\hline$\geq 37$ weeks & $38(25.33)$ \\
\hline \multicolumn{2}{|l|}{ Mode of delivery N (\%) } \\
\hline Spontaneous vaginal delivery & $80(53.33)$ \\
\hline Caesarean section & $64(42.67)$ \\
\hline Assisted delivery & $6(4)$ \\
\hline \multicolumn{2}{|l|}{ Birthplace N (\%) } \\
\hline Inborn (at institute) & $84(56)$ \\
\hline Out born & $66(44)$ \\
\hline \multicolumn{2}{|l|}{ Sepsis type N (\%) } \\
\hline EOS & $91(60.67)$ \\
\hline LOS & $59(39.33)$ \\
\hline
\end{tabular}

EOS- early onset sepsis; LOS-late onset sepsis.

Analysis of the type of neonatal sepsis showed that early onset sepsis (EOS) constituted 60.67\% (95\% CI 50.63\% to $70.71 \%$ ) while $39.33 \%$ (95\% CI $26.86 \%$ to $51.79 \%$ ) were late onset sepsis (LOS). Culture proven sepsis was found in $37.33 \%$ (95\% CI $24.66 \%$ to $50.00 \%$ ) neonates. Culture negative cases were significantly higher in EOS compared to LOS (p value 0.005 ).

The microorganism profile of culture positive sepsis cases is enlisted in (Table 2). The most common pathogen isolated from biological samples of culture proven sepsis were Klebsiella sp (53.57\%); E. coli (10.71\%). CONS, Pseudomonas, and Candida sp had similar frequency of occurrence and Staphylococcus aureus was isolated in $5.36 \%$ of cases.

Table 2: Microorganism profile of culture positive cases.

\begin{tabular}{|c|c|c|}
\hline \multirow[t]{2}{*}{ Organism } & $\begin{array}{l}\text { Total number of } \\
\text { culture positive } \\
\text { cases }(n=56)\end{array}$ & $\begin{array}{l}95 \% \text { CI of the } \\
\text { proportion }\end{array}$ \\
\hline & $\mathrm{N}(\%)$ & \\
\hline Klebsiella sp & $30(53.57)$ & $\begin{array}{l}35.72 \% \text { to } \\
71.42 \%\end{array}$ \\
\hline E. coli & $6(10.71)$ & $\begin{array}{l}-14.03 \% \text { to } \\
35.45 \%\end{array}$ \\
\hline CONS & $4(7.14 \%)$ & $\begin{array}{l}-18.09 \text { to } \\
32.37 \%\end{array}$ \\
\hline Pseudomonas & $4(7.14 \%)$ & $\begin{array}{l}-18.09 \text { to } \\
32.37 \%\end{array}$ \\
\hline Acinetobacter & $3(5.36 \%)$ & $\begin{array}{l}-20.13 \% \text { to } \\
30.85 \%\end{array}$ \\
\hline $\begin{array}{l}\text { Staphylococcus } \\
\text { aureus }\end{array}$ & $3(5.36 \%)$ & $\begin{array}{l}-20.13 \% \text { to } \\
30.85 \%\end{array}$ \\
\hline $\begin{array}{l}\text { Streptococcus } \\
\text { group D }\end{array}$ & $1(1.78 \%)$ & $\begin{array}{l}-24.01 \% \text { to } \\
27.53 \%\end{array}$ \\
\hline Acinetobacter & $1(1.78 \%)$ & $\begin{array}{l}-24.01 \% \text { to } \\
27.53 \%\end{array}$ \\
\hline Candida sp & $4(7.14 \%)$ & $\begin{array}{l}-18.09 \text { to } \\
32.37 \%\end{array}$ \\
\hline
\end{tabular}

CONS- Coagulase negative Staphylococcus aureus.

The antimicrobial agents used are depicted in (Table 3). The most common AMA prescribed was netilmycin $(94.67 \%)$ followed by piperacillin-tazobactam $(88.67 \%)$ and ofloxacin $(29.33 \%)$.

Meropenem was prescribed in $16 \%$ of the study population. Use of reserve antimicrobials like colistin, vancomycin and linezolid was restricted to only $0.67 \%$ cases.

Amphotericin B was the only systemic antifungal prescribed while acyclovir was the sole antiviral drug prescribed. Majority of neonates (58\%) received 2 antimicrobial agents, $39.33 \%$ of cases received $\geq 3$ AMAs and only $2.67 \%$ were administered single AMA.

Overall the days of therapy (DOT) with antimicrobial agent was 3252 with a median of 18 (IQR 14 to 28) days. 
During the study period there were 5289 inpatient days. Antimicrobial consumption described as DOT/1000 PD was 614.86. DOT per neonate was 21.68 (Table 4). The three largest contributors were aminoglycosides, penicillins and fluoroquinolones.

Table 3: List of systemic antimicrobial agents used.

\begin{tabular}{|ll|}
\hline Agent & $\begin{array}{l}\text { Number of neonates who } \\
\text { received at least one } \\
\text { course N }(\%)\end{array}$ \\
\hline Aminoglycosides & $142(94.67)$ \\
\hline Netilmycin & $2(1.33)$ \\
\hline Gentamicin & $4(2.67)$ \\
\hline Amikacin & \\
\hline B lactam antibiotics & $133(88.67)$ \\
\hline $\begin{array}{l}\text { Piperacillin- } \\
\text { Tazobactam }\end{array}$ & $10(6.67)$ \\
\hline $\begin{array}{l}\text { Amoxycillin-clavulanic } \\
\text { acid }\end{array}$ & $1(0.67)$ \\
\hline Ampicillin & $4(2.67)$ \\
\hline Cefotaxime & $1(0.67)$ \\
\hline Ceftizoxime & $24(16)$ \\
\hline Meropenem & \\
\hline Fluroquinolones & $44(29.33)$ \\
\hline Ofloxacin & $1(0.67)$ \\
\hline Ciprofloxacin & $1(0.67)$ \\
\hline Oxazolidinedione & $1(0.67)$ \\
\hline Linezolid & $4(2.67)$ \\
\hline Glycopeptide & \\
\hline Vancomycin & \\
\hline Nitroimidalzole & \\
\hline Metronidazole & \\
\hline Polypeptide & \\
\hline Colistin & \\
\hline $\begin{array}{l}\text { Antifungal } \\
\text { Amphotericin B) }\end{array}$ & \\
\hline Antiviral (Aciclovir) & \\
\hline
\end{tabular}

There was no statistically significant difference in AMA consumption in EOS versus LOS but AMA consumption was significantly higher in culture positive versus negative cases with a $\mathrm{p}$ value of $<0.001$.

Table 4: Quantification of antimicrobial consumption in terms of DOT.

\begin{tabular}{|ll|}
\hline Variable & Value \\
\hline Total DOT & 3252 \\
\hline Total patient days & 5289 \\
\hline DOT/1000 patient days & 614.86 \\
\hline DOT/neonate & 21.68 \\
\hline
\end{tabular}

DOT- days of therapy.

Antimicrobials consumption was also not different between inborn and out born neonates. The results are summarized in Table 6.
Results of univariate analysis for delineating association of different variables with culture positive sepsis revealed that culture negative cases were significantly higher in early onset sepsis compared to late onset sepsis with a $p$ value of 0.005 , while place and mode of delivery, gestational categories were not statistically significant. Table 7 lists the results of the univariate analysis.

Table 5: Quantification of major antimicrobial consumption by classes.

\begin{tabular}{|llll|}
\hline $\begin{array}{l}\text { Antimicrobial } \\
\text { agents }\end{array}$ & DOT & $\begin{array}{l}\text { DOT } \\
\text { per } \\
\text { 1000 } \\
\text { PD }\end{array}$ & $\begin{array}{l}\text { \% } \\
\text { consumption }\end{array}$ \\
\hline Aminoglycosides & 1317 & 249 & 40.50 \\
\hline Penicillins & 1027 & 194.18 & 31.58 \\
\hline Fluoroquinolones & 405 & 76.57 & 12.45 \\
\hline Carbapenems & 258 & 48.78 & 7.93 \\
\hline Cephalosporins & 47 & 8.89 & 1.45 \\
\hline Nitroimidazole & 34 & 6.43 & 1.05 \\
\hline Polypeptide & 21 & 3.97 & 0.64 \\
\hline Oxazolidinone & 14 & 2.56 & 0.43 \\
\hline Glycopeptide & 14 & 2.56 & 0.43 \\
\hline Antifungal & 83 & 15.69 & 2.55 \\
\hline Antiviral & 40 & 7.56 & 1.23 \\
\hline
\end{tabular}

DOT: days of therapy; PD- patient days.

Table 6: Comparison of DOT amongst different sepsis categories.

\begin{tabular}{|c|c|c|c|}
\hline Category & $\begin{array}{l}\text { DOT/1000 } \\
\text { patient days }\end{array}$ & $\begin{array}{l}\text { Total } \\
\text { DOT }\end{array}$ & $\begin{array}{l}\text { P value for } \\
\text { total DOT } \\
\text { comparison }\end{array}$ \\
\hline $\begin{array}{l}\text { EOS }(<72 \\
\text { hours of } \\
\text { age })\end{array}$ & 353.56 & 1870 & \multirow[t]{2}{*}{0.952} \\
\hline $\begin{array}{l}\text { LOS }(\geq 72 \\
\text { hours of } \\
\text { age) }\end{array}$ & 261.29 & 1382 & \\
\hline $\begin{array}{l}\text { Culture } \\
\text { positive }\end{array}$ & 314.62 & 1664 & \multirow[t]{2}{*}{$<0.001$} \\
\hline $\begin{array}{l}\text { Culture } \\
\text { negative }\end{array}$ & 300.24 & 1588 & \\
\hline Inborn & 335.98 & 1777 & \multirow{2}{*}{0.787} \\
\hline Outborn & 278.88 & 1475 & \\
\hline
\end{tabular}

EOS- early onset sepsis; LOS-late onset sepsis *p value $<0.05$ considered significant, test for significance: Mann Whitney U test.

Analysis of treatment outcome showed that majority of neonates $97(64.67 \%)$ were discharged while fatality was observed in $28.67 \%$ cases. $8 \%$ patients were transferred out to other hospitals and $2 \%$ left against medical advice. (Figure 2). 
Univariate analysis for delineating association of different variables with death (Table 8) revealed that survival in case of culture negative sepsis were significantly higher than culture positive cases with a $\mathrm{p}$ value of 0.005 . Other variables failed to show any statistically significant association with death. The outcome of neonatal sepsis is not merely dependent on usage of AMA but other measures like respiratory support, intravenous fluids, phototherapy, temperature and glucose regulation as well as prevention of hospital acquired infections.

Table 7: Univariate analysis of various variables with culture positivity.

\begin{tabular}{|c|c|c|c|c|}
\hline Category & $\begin{array}{l}\text { Culture positive } \\
\text { sepsis }(n=56)\end{array}$ & $\begin{array}{l}\text { Culture negative } \\
\text { sepsis }(n=94)\end{array}$ & P value & $\begin{array}{l}\text { Odds ratio } \\
(95 \% \mathrm{CI})\end{array}$ \\
\hline \multicolumn{5}{|l|}{ Type of sepsis } \\
\hline EOS & 26 & 65 & \multirow{2}{*}{0.005} & \multirow{2}{*}{$0.39(0.19$ to 0.77$)$} \\
\hline LOS & 30 & 29 & & \\
\hline \multicolumn{5}{|c|}{ Place of delivery } \\
\hline Inborn & 27 & 57 & \multirow{2}{*}{0.14} & $0.6(0.31$ to 1.2$)$ \\
\hline Outborn & 29 & 37 & & \\
\hline \multicolumn{5}{|c|}{ Mode of delivery } \\
\hline SVD\& assisted & 38 & 48 & \multirow{2}{*}{0.04} & $2(1$ to 4$)$ \\
\hline LUCS & 18 & 46 & & \\
\hline \multicolumn{5}{|c|}{ Gestational category } \\
\hline$<37$ weeks & 37 & 75 & \multirow{2}{*}{0.06} & $0.49(0.23$ to 1.04$)$ \\
\hline$\geq 37$ weeks & 19 & 19 & & \\
\hline
\end{tabular}

EOS- early onset sepsis; LOS-late onset sepsis; SVD- spontaneous vaginal delivery; LUCS- lower uterine caesarean section. *p value $<0.05$ considered significant, test for significance: Chi square test.

Table 8: Association of variables with death.

\begin{tabular}{|c|c|c|c|c|}
\hline Category & Death $(n=43)$ & Survived (n=107) & P value & Odds ratio $(95 \% \mathrm{CI})$ \\
\hline Culture positive & 24 & 32 & \multirow[t]{2}{*}{0.005} & \multirow[t]{2}{*}{$2.96(1.48$ to 6.15$)$} \\
\hline Culture negative & 19 & 75 & & \\
\hline EOS & 27 & 64 & \multirow[t]{2}{*}{0.85} & \multirow[t]{2}{*}{$1.1(0.55$ to 2.4$)$} \\
\hline LOS & 16 & 43 & & \\
\hline Inborn & 26 & 58 & \multirow[t]{2}{*}{0.59} & \multirow[t]{2}{*}{$1.29(0.63$ to 2.66$)$} \\
\hline Out born & 17 & 49 & & \\
\hline \multicolumn{3}{|c|}{ Gestational category } & \multirow[t]{3}{*}{0.23} & \multirow[t]{3}{*}{$1.7(0.71$ to 4.1$)$} \\
\hline$<37$ weeks & 35 & 77 & & \\
\hline$\geq 37$ weeks & 8 & 30 & & \\
\hline
\end{tabular}

EOS- early onset sepsis; LOS-late onset sepsis.

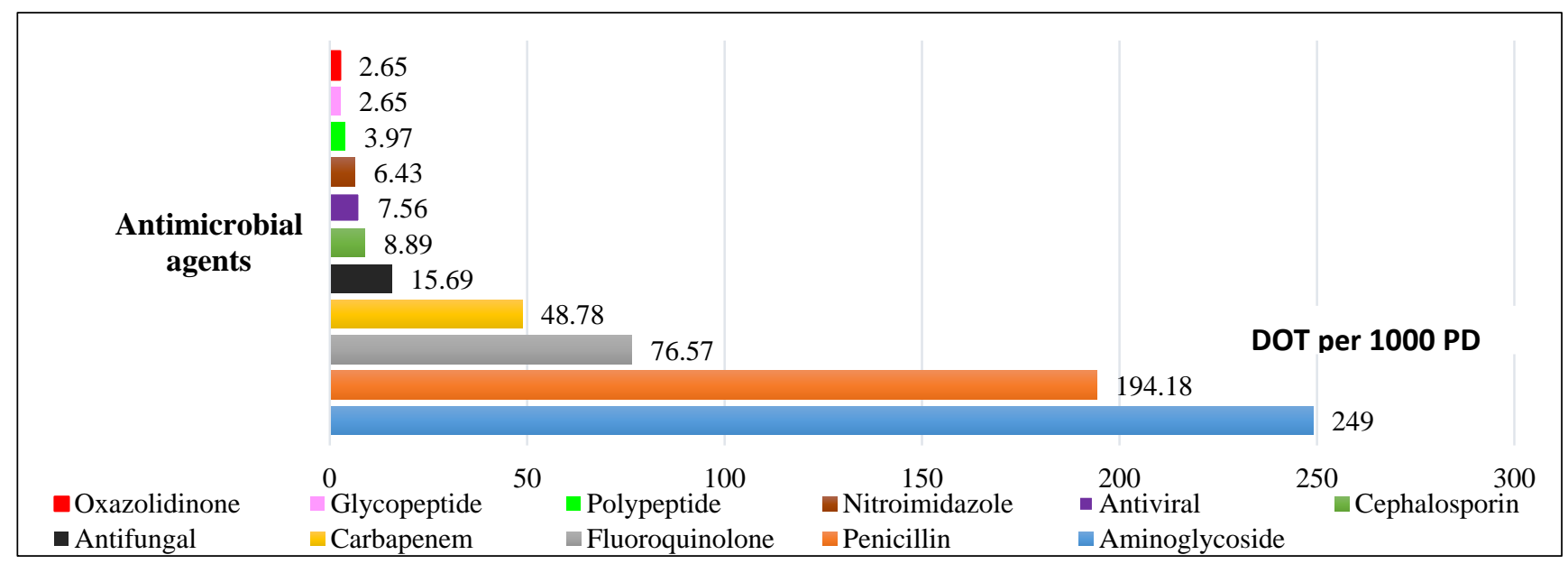

Figure 1: Antimicrobial agents with respect to DOT per 1000 PD. 


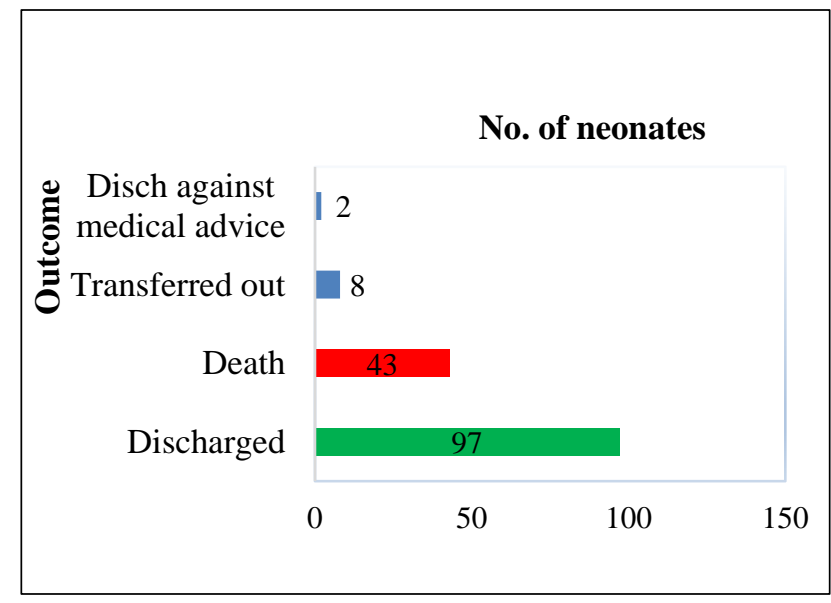

Figure 2: Treatment outcome of enrolled subjects.

\section{DISCUSSION}

Antimicrobial agents are the most commonly used drugs in neonatal intensive care units. Although drug utilization studies give an overview of the antimicrobial prescribing patterns but documentation of the consumption of these agents is considered as a surrogate indicator in any antibiotic stewardship programme. This prospective observational study provides a comprehensive evaluation of antimicrobial usage and its consumption patterns in a level 3 NICU setting of a public tertiary care hospital from an Indian perspective.

Analysis of the antimicrobial prescribing pattern showed that majority of the neonates $(58 \%)$ received 2 antimicrobial agents with $39.33 \%$ of cases received $\geq 3$ AMAs and only $2.67 \%$ were administered single antimicrobial. It was observed that $90.7 \%$ of all study subjects at NICU were treated with antibiotics and mostly two antibiotics. ${ }^{11}$ The most common AMA prescribed in our study was netilmicin $(94.67 \%)$ followed by piperacillin-tazobactam (88.67\%). Third and fourth common AMAs were ofloxacin (29.33\%) and meropenem (16\%) respectively. The local guidelines for first line antimicrobial therapy in NS at the study setting during the study period was an aminoglycoside (netilmicin) and a beta lactam (piperacillintazobactam).There was no difference in usage pattern of AMAs in inborn and out born neonates.

Our study results are comparable to a published study from western India. The study was conducted in 2014 in a NICU of a tertiary care hospital in Western Maharashtra. ${ }^{12}$ The results showed that out of total 528 neonates, 370 received antibiotics. Majority of neonates (55.9\%) received between 1-2 antibiotics, $37.3 \%$ were prescribed 3 to 5 antibiotics. Antibiotics most commonly prescribed were- amikacin, cefotaxime and levofloxacin. Amikacin and cefotaxime were prescribed more commonly to term infants whereas other antibiotics like levofloxacin, piperacillin-tazobactam and meropenem were prescribed more frequently to preterm and out born neonates.

In our study there was no difference in type of AMAs used in term and preterm neonates. Their target population were broader in terms that they included all possible indications for AMA use in NICU whereas our study population included only patients with clinical sepsis.

A detailed Pubmed search showed very few studies from India have quantified AMA consumption in NICU in terms of DOT. Bansal et al measured antimicrobial usage in terms of both DOT and DDD but in adults. ${ }^{13}$ Results from international studies like the SCOUT study done in a level 3C NICU setting in Texas, USA showed that the total antibiotic use was 343.2 DOT/1000 PD and 5.7 DOT/infant. of $72 \%$ of infants who received antimicrobials, $43 \%$ received one course and $29 \%$ more than one course of AMAs. Gentamicin (46\%), ampicillin $(39 \%)$, and oxacillin (8\%) were the most frequently used agents. 94\% of antibiotic use (323 DOT/1000 PD) was empiric therapy for suspected infection. Of antimicrobial class used in terms of DOT per 1000 PD were almost similar to this study. The consumption in culture proven sepsis in their study was only 17.4 DOT per $1000 \mathrm{PD}$, whereas in our study DOT per 1000 PD for culture positive cases was 314.62. This difference may be due to difference in the rate of culture positivity in these two study settings. The same group conducted the study in $2013-14,{ }^{14}$ following an intervention under an antibiotic stewardship program, whereby the total DOT was reduced by $27 \%$ to $252.2 \mathrm{DOT} / 1000 \mathrm{PD}$.

Appropriate identification of neonatal sepsis in the NICU remains a challenge, as they usually present with nonspecific features that overlap with non-infectious etiologies. ${ }^{15,16}$ The need for empiric therapy can only be minimized if clinical findings can be corroborated with early microbiological laboratory testing results. ${ }^{17}$ The choice of antimicrobial regimes mainly depends on information of the flora prevalent in the unit, clinical response and culture reports. In our study there was no statistically significant difference between consumption of AMAs between inborn and referral cases. The usage pattern of AMA for inborn and referral cases is found to be similar in our study.

Suryawanshi et al found in their study that to give wider coverage, as per their antimicrobial policy, the out born neonates received amikacin and cefotaxime as an empirical therapy, while netilmycin and amoxicillinclavulanic acid was common among inborn neonates for both gram positive and gram negative organisms. ${ }^{12}$ Antibiotics were changed to levofloxacin, piperacillin, meropenem or colistin according to culture and sensitivity reports. The use of antimicrobials were more in preterm neonates similar to our study. The use of reserve antibiotics like colistin for multi-drug resistant 
organism was very limited in our study as there was only one case where it was found to be used.

Stewardship efforts must focus on timely discontinuation of antimicrobial therapy once infection is no longer suspected or culture is sterile beyond 48 hours. ${ }^{18} \mathrm{We}$ observed high rate of continuation of AMA beyond 48 hours when culture was negative $(62.67 \%)$. Almost similar results have been reported from other studies. ${ }^{15,19}$ Discontinuation at 48 hours may help in curbing over usage of antimicrobials but will require close monitoring to prevent inadverant discontinuation of needed therapy. ${ }^{20}$

The organism profile of culture positive cases in this study showed that Klebsiella $s p$ was isolated in $53.57 \%$; E.coli in $10.71 \%$, Coagulase negative Staph sp (CONS), Pseudomonas, and Candida sp with similar frequency of occurrence i.e $7.14 \%$. Review of organisms isolated in NS cases across different parts of India was also undertaken. Reports from a study conducted in a similar NICU setting in western India where coagulase negative Staphylococcus, Enterococcus fecalis and Staphylococcus aureus were the most commonly isolated organisms in order of frequency. A retrospective study conducted in NICU in Northern India over a five year period revealed that Staphylococcus aureus (47.3\%), Klebsiella pneumonia (14.9\%) and Acinetobacter (14.9\%) were the most common organisms. ${ }^{21}$ Another study from Western India reported that $26.6 \%$ of NS cases were culture positive and Klebsiella pneumoniae was the most common gram negative organism (35.4\%) and Staphylococcus aureus $(22.9 \%)$ was the predominant gram-positive pathogen. ${ }^{22}$

The limitations of our study include those that are inherent of any observational study. The data collected from out born patients was often incomplete mainly with respect to the antimicrobial intake history prior to admission. Several out born neonates were already on antibiotics prior to admission and the initial blood culture samples were sent after initiation of antimicrobial therapy. Finally, though this study spanned for over 18 month our study results may not be generalized to all NICU settings due to variability in the organism profile implicated in the causation of NS. In order to reduce inappropriate antimicrobial use in NS, further efforts need to be taken to sensitize prescribers to de-escalate or discontinue antimicrobials in culture negative clinically stable cases which serves as an important tenet in the advocacy of antimicrobial stewardship.

\section{ACKNOWLEDGEMENTS}

Prior presentations at oral presentation at the Annual conference of the Indian Pharmacological Society Conference at Patna in February 2017. We are thankful to Dr. Jagdish Sahoo, former DMPGT of the Department Neonatology and Mr. Deepak Singh, Mr. Asim Sutar, technical staff of NICU for assistance in microbiology laboratory data collection. We are also grateful to other postdoctoral trainees, faculty members and nursing staff of the Department of Neonatology for assisting in study data collection.

Funding: No funding sources

Conflict of interest: None declared

Ethical approval: The study was approved by the Institutional Ethics Committee

\section{REFERENCES}

1. Report of the National Neonatal Perinatal Database (National Neonatology Forum). 2002-03; 1-13.

2. Thaver D, Zaidi AK. Burden of neonatal infections in developing countries: a review of evidence from community-based studies. Pediatric Infectious Dis J. 2009 Jan 1;28(1):S3-9.

3. Bang AT, Bang RA, Baitule SB, Reddy MH, Deshmukh MD. Effect of home-based neonatal care and management of sepsis on neonatal mortality: field trial in rural India. Lancet. 1999;354(9194):1955-61.

4. Stoll BJ. The global impact of neonatal infection. Clin Perinatol. 1997;24(1):1-21.

5. Clark RH, Bloom BT, Spitzer AR, Gerstmann DR. Reported medication use in the neonatal intensive care unit: data from a large national data set. Pediatr. 2006;117(6):1979-87.

6. Cantey JB, Wozniak PS, Sánchez PJ. Prospective surveillance of antibiotic use in the neonatal intensive care unit: results from the SCOUT study. Pediatr Infect Dis J. 2015;34(3):267-72.

7. Cantey JB, Sánchez PJ. Prolonged antibiotic therapy for culture-negative sepsis in preterm infants: it's time to stop. J Pediatr. 2011;159(5):707-8.

8. Ibrahim OM, Polk RE. Antimicrobial use metrics and benchmarking to improve stewardship outcomes: methodology, opportunities, and challenges. Infect Dis Clin North Am. 2014;28(2):195-214.

9. Van der Meer JW, Gyssens IC. Quality of antimicrobial drug prescription in hospital. Clin Microbiol Infect. 2001;6:12-5.

10. Society for Healthcare Epidemiology of America, Infectious Diseases Society of America, Pediatric Infectious Diseases Society. Policy statement on antimicrobial stewardship by the Society for Healthcare Epidemiology of America (SHEA), the Infectious Diseases Society of America (IDSA), and the Pediatric Infectious Diseases Society (PIDS). Infect Control Hosp Epidemiol. 2012;33(4):322-7.

11. Neubert A, Lukas K, Leis T, Dormann H, Brune K, Rascher W. Drug utilisation on a preterm and neonatal intensive care unit in Germany: a prospective, cohort-based analysis. Eur J Clin Pharmacol. 2010;66(1):87-95.

12. Suryawanshi S, Pandit V, Suryawanshi P, Panditrao A. Antibiotic Prescribing Pattern in a Tertiary Level Neonatal Intensive Care Unit. J Clin Diagn Res. 2015;9(11):21-4. 
13. Bansal D, Mangla S, Undela K, Gudala K, D’Cruz S, Sachdev A, et al. Measurement of adult antimicrobial drug use in tertiary care hospital using defined daily dose and days of therapy. Indian $\mathbf{J}$ Pharm Sci. 2014;76(3):211-7.

14. Cantey JB, Wozniak PS, Pruszynski JE, Sánchez PJ. Reducing unnecessary antibiotic use in the neonatal intensive care unit (SCOUT): a prospective interrupted time-series study. Lancet Infect Dis. 2016;16(10):1178-84.

15. Fischer JE. Physicians' ability to diagnose sepsis in newborns and critically ill children. Pediatr Crit Care Med. 2005;6(3):120-5.

16. Bekhof J, Reitsma JB, Kok JH, Van Straaten IHLM. Clinical signs to identify late-onset sepsis in preterm infants. Eur J Pediatr. 2013;172(4):501-8.

17. Escobar GJ, Puopolo KM, Wi S, Turk BJ, Kuzniewicz MW, Walsh EM, et al. Stratification of risk of early-onset sepsis in newborns $\geq 34$ weeks' gestation. Pediatrics. 2014;133(1):30-6.

18. Patel SJ, Oshodi A, Prasad P, Delamora P, Larson E, Zaoutis T, et al. Antibiotic use in neonatal intensive care units and adherence with Centers for Disease Control and Prevention 12 Step Campaign to Prevent Antimicrobial Resistance. Pediatr Infect Dis J. 2009;28(12):1047-51.
19. Shipp K, Chiang T, Karasick S, Quick K, Nguyen S, Cantey J. Antibiotic Stewardship Challenges in a Referral Neonatal Intensive Care Unit. Am J Perinatol. 2016;33(5):518-24.

20. Jardine MA, Kumar Y, Kausalya S, Harigopal S, Wong J, Shivaram A, et al. Reducing antibiotic use on the neonatal unit by improving communication of blood culture results: a completed audit cycle. Arch Dis Child Fetal Neonatal Ed. 2003;88(3):255.

21. Marwah P, Chawla D, Chander J, Guglani V, Marwah A. Bacteriological profile of neonatal sepsis in a tertiary-care hospital of Northern India. Indian Pediatr. 2015;52(2):158-9.

22. Muley VA, Ghadage DP, Bhore AV. Bacteriological Profile of Neonatal Septicemia in a Tertiary Care Hospital from Western India. J Glob Infect Dis. 2015;7(2):75-7.

Cite this article as: Kumari M, Chatterjee S, Som T Chattopadhyay P. Antimicrobial usage and its quantification for neonatal sepsis at a tertiary care hospital neonatal intensive care unit. Int J Basic Clin Pharmacol 2019;8:2046-53. 